\title{
Advances in vaccine adjuvants
}

\author{
Manmohan Singh* and Derek O’Hagan \\ Chiron Corporation, 5300 Chiron Way, Emeryville, CA 94608. ${ }^{\star}$ Corresponding author (Manmohan_Singh@cc.chiron.com).
}

Received 8 December 1998; accepted 20 September 1999

\begin{abstract}
Currently, aluminum salts and MF59 are the only vaccine adjuvants approved for human use. With the development of new-generation vaccines (including recombinant subunit and mucosal vaccines) that are less immunogenic, the search for more potent vaccine adjuvants has intensified. Of the novel compounds recently evaluated in human trials, immunostimulatory molecules such as the lipopolysaccharide derived MPL and the saponin derivative QS21 appear most promising, although doubts have been raised as to their safety in humans. Preclinical work with particulate adjuvants, such as the MF59 microemulsion and lipid-particle immune-stimulating complexes (Iscoms), suggest that these molecules are also potent elicitors of humoral and cellular immune responses. In addition, preclinical data on CpG oligonucleotides appear to be encouraging, particularly with respect to their ability to selectively manipulate immune responses. While all these adjuvants show promise, further work is needed to better define the mechanisms of adjuvant action. Ultimately, the development of more potent adjuvants may allow vaccines to be used as therapeutic, rather than prophylactic, agents.
\end{abstract}

Keywords: adjuvant, recombinant vaccine delivery, mucosal delivery, vaccine targeting

Vaccines have traditionally consisted of live attenuated pathogens, whole inactivated organisms, or inactivated toxins. Although these have proved successful in the past, several drawbacks have limited their use against more challenging diseases such as hepatitis $\mathrm{C}$ or AIDS. First, certain live-attenuated vaccines can cause disease in immunosuppressed individuals by reverting to a more virulent phenotype. Second, whole inactivated vaccines (e.g., Bordetella pertussis) contain reactogenic components that can cause undesirable side effects. Third, some pathogens are difficult or even impossible to grow in culture (e.g., hepatitis $\mathrm{B}$, hepatitis $\mathrm{C}$, and human papillomavirus), making preparation of a vaccine problematic.

In the past decade, several new approaches to vaccine development have emerged that may have significant advantages over traditional approaches. These new approaches include recombinant protein subunits, synthetic peptides, and plasmid DNA. Although they offer advantages such as reduced toxicity, they are poorly immunogenic when administered alone. This is particularly true for vaccines based on recombinant proteins or peptides. Traditional vaccines are heterogeneous and contain many epitopes, some of which can provide additional T-cell help or function as adjuvants (e.g., bacterial DNA in whole-cell vaccines). Therefore, a great need exists for immunological adjuvants that are potent, safe, and compatible with new-generation vaccines, including DNA vaccines.

Immunological adjuvants were originally described by Ramon ${ }^{1}$ as "substances used in combination with a specific antigen that produce more immunity than the antigen alone." This broad definition encompasses a very wide range of materials ${ }^{2}$. However, despite extensive evaluation over many years, the only adjuvants currently approved by the US Food \& Drug Administration (Rockville, MD) are aluminum-based mineral salts (generically called alum). Alum has a good safety record, but comparative studies show that it is a weak adjuvant for antibody induction to protein subunits and a poor adjuvant for cell-mediated immunity ${ }^{3}$. Moreover, alum adjuvants can induce immunoglobulin E (IgE) antibody responses and have been associated with some allergic reactions in human subjects ${ }^{3,4}$.

The most important issue in adjuvant development is safety, which has restricted the development of adjuvants since alum was first introduced more than 50 years ago $0^{5}$. Many experimental adjuvants have advanced to clinical trials and some have demonstrated high potency, but most have proved too toxic for routine clinical use. For standard prophylactic immunization in healthy individuals, only adjuvants that induce minimal side effects will prove acceptable. In contrast, for adjuvants that are designed to be used in life-threatening situations (e.g., cancer), the acceptable level of adverse events is likely to be higher. Additional issues that are important for adjuvant development include biodegradability, stability, ease of manufacture, cost, and applicability to a wide range of vaccines. Ideally, for ease of administration and patient compliance, an adjuvant should allow a vaccine to be given by a mucosal route. Examples of adjuvants that have been evaluated in clinical trials are shown in Table 1. Although the mechanisms of action of adjuvants are often poorly understood ${ }^{2,5}$, they can be broadly classified on the basis of their principal mode of action. However, these classifications become more arbitrary as immunostimulatory adjuvants are added to vaccine delivery systems and adjuvants are combined in single formulations.

\section{The role of adjuvants in vaccine development}

Adjuvants can be used to improve the immune response to vaccine antigens in many different ways: they can increase the immunogenicity of weak antigens; they can be used to enhance the speed and duration of the immune response; they can modulate antibody avidity, specificity, isotype, or subclass distribution; they can stimulate cell-mediated immunity; they can promote the induction of mucosal immunity; they can enhance immune responses in immunologically immature, or senescent individuals; they can decrease the dose of antigen and reduce vaccine costs; or they can help overcome antigen competition in combination vaccines.

The mechanisms of adjuvant action are currently only poorly understood, which has hampered rational design of new adjuvant compounds. Immunization often activates several complex cascades of immune effectors, only some of which are relevant to the induction of an antigen-specific response. Often, ascertaining the exact effectors enhanced by a particular adjuvant is difficult to clearly define in vivo. 
REVIEW

Table 1. Selected examples of vaccine adjuvants ${ }^{a}$

Mineral salts

Mucosal adjuvants

Lipid particles

Microparticulate adjuvants

MDP derivatives

CpG oligos

LPS

MPL

Liposomes

Virosomes*

Iscoms

Cochleates

Virus-like particles

Cholera toxin (CT)

Microparticles
Aluminum hydroxide*

Aluminum phosphate*

Calcium phosphate

Cytokines (e.g., IL-2, IL-12, GM-CSF)

Saponins (e.g., QS21)

polyphosphazenes

Emulsions (e.g., Freund's, SAF, MF59*)

PLG microparticles

Poloxamer particles

Heat-labile enterotoxin (LT)

Mutant toxins (e.g., LTK63 and LTR72)

Polymerized liposomes

awith the exception of CpGs, cochleates, and polymerized liposomes, all of these adjuvants have been evaluated in clinical trials. However, only those marked with an asterisk are currently included as adjuvants in approved vaccine products for human use.

That said, adjuvants can mediate their effects by any of the following mechanisms: increasing cellular infiltration, inflammation, and trafficking to the injection site-particularly for antigen-presenting cells (APCs); promoting the activation state of APCs by upregulating costimulatory signals or major histocompatibility complex (MHC) expression; enhancing antigen presentation; or inducing cytokine release for indirect effects. The most appropriate adjuvant for a given vaccine antigen will depend to a large extent on the type of immune response that is required for protective immunity. However, for many infectious diseases, the types of responses required for protective immunity have yet to be established. Unfortunately, in these situations, adjuvant selection becomes somewhat empirical.

A simplified representation of the immune response to an injected vaccine antigen is shown in Figure 1. Two major subsets of $\mathrm{CD}^{+} \mathrm{T}$ cells (T-helper 1 [Th1] and Th2) have been identified in mouse and human, based on their secretion of different cytokine patterns $s^{6-8}$. Th1 responses are typically characterized by the induction of delayed-type hypersensitivity responses, and secretion of interferon- $\gamma($ IFN- $\gamma$ ), interleukin 2 (IL-2), and IL-12. In contrast, Th2 responses are characterized by the induction of circulating or secretory antibodies and the secretion of IL-4, IL-5, IL-6, and IL-10. The different cytokine secretion patterns are mutually antagonistic, and upregulation of one type of response normally results in downregulation of the alternative. In mice, the production of the IgG2a antibody isotype is widely recognized as characteristic of a Th1 response ${ }^{9}$, whereas a Th2 response is associated with the induction of IgG1.

Although different adjuvants may induce comparable levels of functional antibodies, the respective cytokine profiles and antibody isotypes may differ (M. Singh, D. O'Hagan, unpublished data). In certain situations, the type of response induced (Th1 or Th2) may have a significant impact on the protective efficacy of a vaccine. Alternative adjuvants do tend to favor specific types of responses, and this may provide some degree of guidance in adjuvant selection. However, adjuvant choice is complicated by commercial constraints and availability. In addition, some adjuvants need to be manufactured using specialized techniques and equipment that are not generally available or suitable for all antigens (e.g., see Iscoms below).

\section{Immunostimulatory adjuvants}

Immunostimulatory adjuvants are thought to exert their effects predominantly at the cytokine level through the activation of MHC molecules, through costimulatory signals, or through related intracellular signaling pathways. One class of immunostimulatory adjuvants is derived from the lipopolysaccharide (LPS) of Gram-negative bacteria. The most extensively evaluated member of this family, monophosphoryl Lipid A (MPL), is obtained from Salmonella minnesota. Although the mechanism of action of MPL has not been clearly defined, it is likely to be similar to LPS. Recent evidence suggests that LPS-mediated immune activation occurs as a consequence of the interaction of LPS with toll-like receptors (TLR), following binding to soluble serum factors, including CD14 ${ }^{10-12}$. Human TLR2 is involved in activation by LPS ${ }^{11,12}$ and triggers cells to produce proinflammatory cytokines via NF- $\kappa \mathrm{B}$, an important element of the response of the innate immune system to infection.

In several preclinical studies, MPL has been shown to induce the synthesis and release of cytokines, particularly IFN$\gamma$, which promotes the generation of Th1 responses ${ }^{13,14}$. As a single adjuvant, MPL does not appear to be very potent for antibody induction, although it appears effective for the induction of $\mathrm{CD}^{+} \mathrm{T}$-cell mediated immunity. Clinically, MPL has often been used in complex formulations, including liposomes and emulsions, and has also been used in adjuvant combinations with alum and QS21 ${ }^{15}$. One problem with such combination studies is that it is difficult to discriminate the contribution of MPL to the overall adjuvant effect. Not only has MPL been clinically evaluated in more than 10,000 subjects ( $\mathrm{T}$. Ulrich, personal communication) combined with

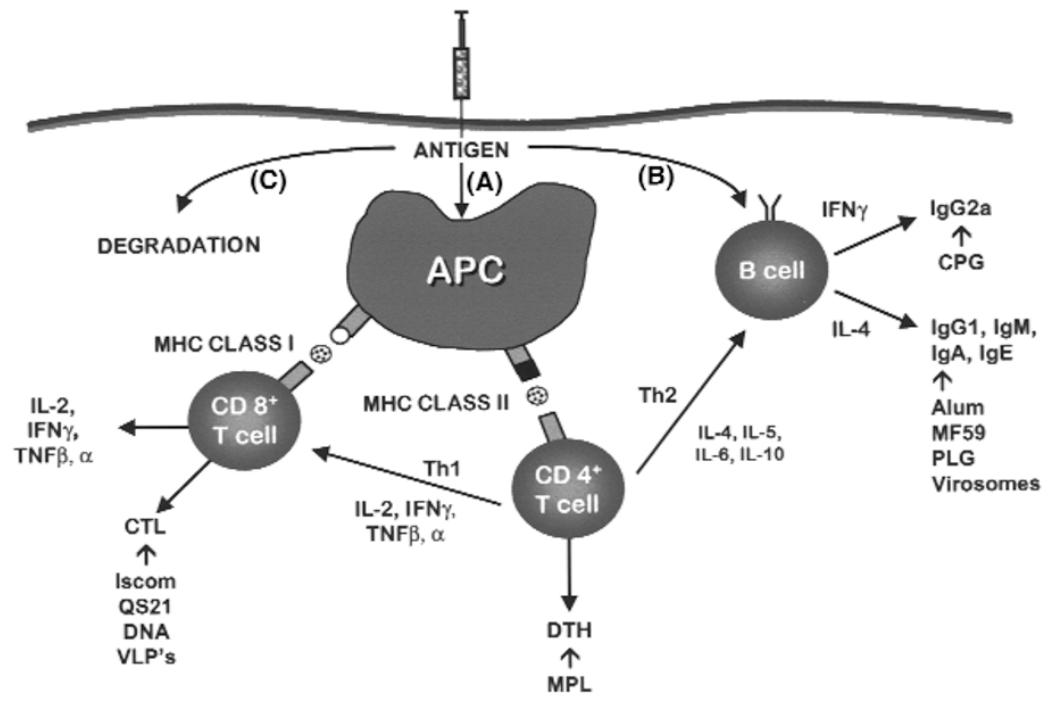

Figure 1. Following injection, vaccine antigens may (A) be directly taken up by antigen-presenting cells (APC), (B) bind to the surface antibody on B cells, or (C) undergo degradation. The pathway taken is largely dependent on the characteristics of the antigen, but may also be influenced by the presence of adjuvants. Antigen taken up by APCs is processed into peptide epitopes and directed through two pathways to MHC molecules (class I and II), which present peptide for interaction with either CD8 ${ }^{+}$or $\mathrm{CD}^{+} \mathrm{T}$ cells, respectively. The stimulated $\mathrm{T}$ cells may secrete cytokines to upregulate the immune response, or act as cytotoxic $\mathrm{T}$ lymphocytes (CTL). Antibodies are produced by B cells with help provided by the cytokines produced by the $\mathrm{CD4}^{+} \mathrm{T}$ cells. In this figure, the main element of the immune response that is stimulated by different adjuvants is indicated by the presence of the respective adjuvants in association with the specific responses. 
vaccines for both cancer (melanoma and breast) and infectious disease vaccines (herpes, hepatitis B virus, malaria, or human papilloma virus), with an acceptable profile of adverse effects, but it has also been used as an adjuvant for DNA vaccines ${ }^{16}$.

A second group of immunostimulatory adjuvants are the triterpenoid glycosides, or saponins, derived from Quillaja saponaria (Chilean soap bark tree). Saponins have been used as adjuvants for many years in veterinary vaccines. However, they are surface-active agents and cause hemolysis of red blood cells in vitro, although hemolysis does not appear to correlate with adjuvant activity ${ }^{17}$. In 1995, Kensil and colleagues ${ }^{18}$ isolated a pure fraction of Quil A saponin with low toxicity (QS21) and defined the structural moieties responsible for adjuvant activity. QS21 has been shown to be a potent adjuvant for cytotoxic T-lymphocyte (CTL) induction, inducing Th1 cytokines (IL-2 and IFN- $\gamma$ ) and antibodies of the IgG2a isotype ${ }^{17}$.

Saponins have been shown to intercalate into cell membranes, through interaction with cholesterol, forming pores ${ }^{19}$. Although it is not known whether the adjuvant effect of saponins is related to pore formation, this seems a likely means to allow antigens to gain access to the cytoplasm for CTL induction. A number of clinical trials have been performed using QS21 as an adjuvant, initially for cancer vaccines (melanoma, breast, and prostate) and subsequently for infectious diseases (HIV-1, influenza, herpes, malaria, or hepatitis B $)^{20}$. Doses of $200 \mu \mathrm{g}$ or higher of QS21 have been associated with significant local reactions $s^{15}$, but lower doses appear to be better tolerated. More than 1,600 volunteers have been immunized with QS21-containing vaccines, the most common side effect being pain/tenderness at the injection site, which is dose related and usually of short duration ${ }^{20,21}$.

QS21 is likely to prove optimally effective as an adjuvant for vaccines against pathogens that require a potent CTL response as an important component of protective immunity. However, the balance of potency versus adverse events is key for this adjuvant, and an effective adjuvant dose that is tolerable needs to be established in humans for each vaccine indication. QS21 has also been used as an adjuvant for DNA vaccines, following both systemic and mucosal administration $^{22}$

Bacterial DNA, but not vertebrate DNA, also has direct immunostimulatory effects on immune cells in vitro ${ }^{23,24}$. The immunostimulatory effect is due to the presence of unmethylated CpG dinucleotides ${ }^{25}$, which are underrepresented and methylated in vertebrate $\mathrm{DNA}^{26}$. Unmethylated $\mathrm{CpGs}$ in the context of selective flanking sequences are thought to be recognized by cells of the immune system to allow discrimination of pathogen-derived DNA from self DNA ${ }^{26}$. Bacterial DNA and CpGs trigger cells of the innate immune system, including macrophages and dendritic cells (DCs), to upregulate MHC class II and costimulatory molecules, transcribe cytokine mRNAs, and secrete proinflammatory cytokines ${ }^{27}$.

The exact mechanism of cellular uptake and activation for CpGs remains unclear, although nonspecific endocytosis may be involved and endosomal maturation appears necessary for the activation of stress kinase pathways, resulting in the release of proinflammatory cytokines $^{28}$. Hence, CpGs bring about conversion of immature DCs into mature APCs and therefore represent a promising new adjuvant ${ }^{29}$. $\mathrm{CpG}$ is most potent for the induction of Th1 responses, mainly through stimulating tumor necrosis factor $\alpha$ (TNF $\alpha)$, IL-1, IL-6, and IL-12, and through the expression of costimulatory molecules ${ }^{29,30}$.

CpGs also appear to have significant potential as mucosally administered adjuvants $^{31,32}$. Their adjuvant effect appears to be maximized by conjugation to protein anti-

\section{Table 2. A comparison}

\begin{tabular}{llll}
\hline Natural pathogens & \multicolumn{2}{l}{ Particulate adjuvants } \\
\hline Bacteria & $\sim 0.5-3 \mu \mathrm{m}$ & Microparticles & $100 \mathrm{~nm}-10 \mu \mathrm{m}$ \\
Poxvirus & $\sim 250 \mathrm{~nm}$ & Liposomes & \\
Herpesvirus & & Virosomes & $50 \mathrm{~nm}-10 \mu \mathrm{m}$ \\
HIV & $\sim 100 \mathrm{~nm}$ & MF59 & $\sim 200 \mathrm{~nm}$ \\
Influenza virus & & & \\
Poliovirus & $20-30 \mathrm{~nm}$ & Iscoms & $\sim 40 \mathrm{~nm}$ \\
Parvovirus & & Virus-like particles & $\sim 20-50 \mathrm{~nm}$
\end{tabular}

gens $^{33}$. Importantly, CpGs also appear to have significant potential for the modulation of existing immune responses, which may be useful in various clinical settings, including allergies ${ }^{34}$. Nevertheless, to date, CpGs have mainly been evaluated only in rodent models and with murine cells; thus their potency and safety in humans remains to be established. Although it is too early to know in which situations CpGs might prove most advantageous, their apparent ability to selectively manipulate immune responses is intriguing.

As an alternative to the use of cytokine-inducing adjuvants, cytokines may also be used directly. Most cytokines have the ability to modify and redirect the immune response. Molecules that have been evaluated most extensively as adjuvants include IL-1, IL-2, IFN- $\gamma$, IL-12, and granulocyte-macrophage colony-stimulating factor $(\mathrm{GM}-\mathrm{CSF})^{35}$. All of these molecules exhibit dose-related toxicity and, because of their proteinaceous nature, have stability problems, a short in vivo half-life, and a relatively high cost of manufacture. Therefore, it is unlikely that cytokines will ever prove feasible as adjuvants in routine vaccination. Nevertheless, considerable progress has been made in the use of cytokines for the immunotherapy of cancer ${ }^{36}$.

\section{Particulate adjuvants}

The use of particulate adjuvants as alternatives to immunostimulatory adjuvants has been evaluated by several groups. Particulate adjuvants (e.g., emulsions, microparticles, Iscoms, liposomes, virosomes, and virus-like particles [VLPs]) have comparable dimensions to the pathogens that the immune system evolved to combat (Table 2). Therefore, particulate adjuvants are naturally targeted for uptake by APCs to facilitate the induction of potent immune responses. Immunostimulatory adjuvants may also be included to enhance the level of immune activation, or to focus the response through a desired pathway (e.g., Th1 or Th2).

In the 1980s, a potent oil-in-water (o/w) adjuvant formulation $(\mathrm{SAF})^{37}$ was developed using a biodegradable oil (squalane). This proved too toxic for widespread use in humans, however, because of the presence of a muramyl dipeptide (MDP) derivative ${ }^{5}$. Subsequently, a squalane o/w emulsion has been developed (MF59) without the presence of additional immunostimulators, and this formulation has proved to be a potent adjuvant with an acceptable safety profile ${ }^{38,39}$. MF59 enhances the immunogenicity of influenza vaccine ${ }^{40-42}$ and has been shown to be a more potent adjuvant than alum for hepatitis B vaccine in baboons ${ }^{43}$. MF59 has also been shown to be an effective adjuvant for a polysaccharide-protein conjugate in infant baboons ${ }^{44}$. Studies with labeled MF59 have confirmed the ability of the adjuvant to target macrophages and dendritic cells, both at the site of injection and in local lymph nodes ${ }^{45}$.

Experience in the clinic (to date, $>30,000$ subjects have been immunized) with several vaccines (including HIV, herpes simplex virus, cytomegalovirus, hepatitis B virus and influenza) has shown that MF59 is safe and well tolerated in humans ${ }^{46,47}$. MF59 was judged to be sufficiently safe to allow the vaccination of newborn infants in a HIV vaccine trial. In summary, MF59 is an effective adjuvant in humans and can be recommended for the induction of potent antibody responses. It has recently been included in a licensed vaccine 
product in Europe and is expected to be included in additional vaccines under development.

Oil/water emulsions have also been used as delivery systems for immunostimulatory adjuvants (MPL and QS21) in a malaria vaccine trial. This approach allowed immunostimulatory adjuvants to be targeted for enhanced uptake by APCs. The level of protection induced in mice by the adjuvants was comparable to, or better than, the levels of protection induced with the Plasmodium yoelii antigen in Freund's complete adjuvant, which, though potent, is too toxic for human use ${ }^{48}$. One adjuvant formulation subsequently showed protective efficacy against an experimental challenge in human volunteers with infected mosquitoes (Plasmodium falciparum) ${ }^{49}$. Nevertheless, the safety of this adjuvant formulation in humans remains to be further evaluated.

Another class of particulate compounds, the liposomes, has been evaluated both as adjuvants and as delivery systems for antigens and adjuvant $s^{50,51}$. It has been difficult to determine their contribution to the overall adjuvant effect because they are often used in complex formulations, for example when in combination with MPL. Nevertheless, a liposomal hepatitis A vaccine (virosome), without additional immunostimulators, has been extensively evaluated in the clinic and is currently undergoing commercial development in Europe $^{52}$. In addition, a virosome-based influenza vaccine has already been introduced onto the market. Modified liposomal structures termed "cochleates" are also being evaluated as systemic and mucosal adjuvants in small-animal models ${ }^{53}$. The development of polymerized liposomes, which show enhanced stability in the gut, also offers potential for the development of mucosal vaccines ${ }^{54}$.

The immunostimulatory fractions from Q. saponaria (Quil A) have been incorporated into lipid particles, comprising cholesterol, phospholipids, and cell membrane antigens termed Iscoms ${ }^{55}$. The mechanism of activity of the adjuvant Quil A is thought to be very similar to QS21, which, as previously discussed, is a purified single fraction from Quil A. In a study in macaques, an influenza Iscom vaccine was more immunogenic than a classical subunit vaccine and induced enhanced protective efficacy ${ }^{56}$. A similar formulation is currently being evaluated in a human clinical trial.

The principal advantage of the preparation of Iscoms is to allow a reduction in the dose of the hemolytic Quil A adjuvant and to target the formulation directly to APCs. A recent study has indicated that the adjuvant effect of Iscoms is mediated by the induction of IL-1257. At present, Iscoms are generally considered to be the most potent adjuvant for the induction of CTL responses in preclinical models. However, their efficacy and safety needs to be established in humans. An added problem with Iscoms is that inclusion of antigens into the adjuvant is often difficult, and may require extensive modification for many antigens.

An alternative approach to target antigens to APCs is represented by the synthesis of biodegradable polymeric microparticles, or the use of polymers or proteins that self-assemble into particulates.

The biodegradable and biocompatible polyesters, poly(lactideco-glycolides) (PLGs), are the primary candidates for the development of microparticles as adjuvants, as they have been used in humans for many years as suture material and as controlled-release delivery systems $\mathrm{s}^{58,59}$. However, the adjuvant effect achieved through the encapsulation of antigens into PLG microparticles has been demonstrated only relatively recently ${ }^{60-63}$. Microparticles appear to mediate their effects largely as a consequence of their uptake into DCs, macrophages, and local lymph nodes following intramuscular injection (M. Dupuis, unpublished data). In contrast to alum, PLG microparticles are effective for the induction of CTL responses in rodents ${ }^{64-66}$. Microparticles also appear to have significant potential as an adjuvant for DNA vaccines ${ }^{67}$.

A particularly attractive feature of microparticles is their ability to control the rate of release of entrapped antigens ${ }^{68,69}$. Controlled release of antigen may allow the development of single-dose vaccines, which would result in improved vaccine compliance, particularly in the developing world, an area where PLG microparticles probably have their greatest long-term potential. However, much more work is needed on the issue of the stability of antigens in microparticles. Alternative polymers that self-associate into particulates (poloxamers) ${ }^{70}$, or soluble polymers (polyphosphazenes) ${ }^{71}$ may also be used as adjuvants, but the safety of these compounds remains to be further evaluated. The adjuvant effect of polyphosphazene polymers has recently been reported in a clinical trial ${ }^{72}$.

Recombinant proteins that naturally self-assemble into particulates can also enhance delivery of antigens to APCs. The first recombinant protein vaccine that was developed, based on hepatitis B surface antigen (HBsAg), was expressed in yeast as a particulate ${ }^{73}$. Recombinant HBsAg is potently immunogenic and can be used to prime CTL responses in vivo ${ }^{74}$. HBsAg and other VLPs can also be used as adjuvants for coexpressed proteins. For example, recombinant Ty VLPs from Saccharomyces cerevisiae carrying a string of up to 15 CTL epitopes from Plasmodium species have been shown to prime protective CTL responses in mice following a single immunization ${ }^{75}$. In addition, Ty VLPs have also been shown to induce CTL activity in macaques against coexpressed simian immunodeficiency virus (SIV) $\mathrm{p} 27^{76}$. Clinical trials of Ty VLPs have shown them to be safe and immunogenic in humans ${ }^{77}$. Nevertheless, the scale-up, manufacture, and purification of VLPs as adjuvants presents formidable difficulties.

\section{Alternative routes of immunization}

Although most vaccines have traditionally been administered by intramuscular injection, mucosal administration of vaccines offers several important advantages. These include easier administration, reduced side effects, and the potential for frequent boosting. In addition, local immunization induces mucosal immunity at the sites where the majority of pathogens initially establish infection of hosts. Oral immunization would be particularly advantageous in isolated communities, where access to health care professionals is difficult. Moreover, this route of immunization would avoid the potential problem of infection due to the re-use of needles. A wide range of approaches are currently being evaluated for the mucosal delivery of vaccines ${ }^{78}$.

In mice, oral immunization with PLG microparticles has been shown to induce potent mucosal and systemic immunity to entrapped antigens ${ }^{79-82}$. Furthermore, mucosal immunization with microparticles induced protection against challenge with Bordetella pertussis ${ }^{83-85}$, Chlamydia trachomatis ${ }^{86}$, and Salmonella typhimuri$u m^{87}$. In primates, mucosal immunization with inactivated SIV in microparticles induced protective immunity against intravaginal challenge ${ }^{88}$. Also in primates, mucosal immunization with microparticles induced protection against aerosol challenge with staphylococcal enterotoxin $\mathrm{B}^{89}$.

Comparative studies have indicated that microparticles are one of the most potent adjuvants available for mucosal delivery of vaccines $^{90}$. In recent studies, microparticles also have shown some promise for the oral delivery of DNA ${ }^{91,92}$. However, optimal responses are likely to be achieved with microparticle formulations that have been modified to maintain the integrity of entrapped $\mathrm{DNA}^{93}$. The ability of microparticles to perform as effective adjuvants following mucosal administration is largely a consequence of their uptake into the specialized mucosal associated lymphoid tissue (MALT) ${ }^{94}$. While microparticles have significant potential for mucosal delivery of vaccines, their potency may be improved by their combination with additional adjuvants.

Many alternative adjuvants have been evaluated for mucosal delivery of vaccines, including the toxins from Vibrio cholerae (CT) and Escherichia coli (LT), which are the most potent mucosal adjuvants available. However, since CT and LT are too toxic for use in 
humans, they have been genetically manipulated to reduce toxicity ${ }^{95-97}$. Single amino acid substitutions in the enzymatic A subunit of LT allowed the development of mutant toxins that retained potent adjuvant activity, but showed negligible or dramatically reduced toxicity ${ }^{98-100}$. Recent studies have shown that LT mutants can induce protective immunity in mice against challenge with Helicobacter pylori ${ }^{101}$. Moreover, intranasal delivery of HIV-1 p24 gag with a nontoxic LT mutant induced potent CTL activity in mouse splenocytes. In contrast, several alternative adjuvants were ineffective for CTL induction in the same study (M. Singh, D. O'Hagan, unpublished data). Although the mechanisms of action of CT and LT mutants remain to be fully defined, it appears that there are important contributions to the adjuvant effect from the $\mathrm{B}$ binding domain, from the presence of an intact A subunit, which interacts with regulatory proteins inside cells, and also from the enzymatic activity of the A1 subunit ${ }^{102}$.

Recent studies have indicated that potent mucosal adjuvants such as CT may also allow vaccination following topical application to the skin ${ }^{103}$. In another recent development, mucosal immunization may also be achieved through the ingestion of transgenic plants expressing antigens and adjuvants ${ }^{104}$.

\section{Application to therapeutic vaccines}

It seems likely that novel adjuvants may prove sufficiently potent to allow the development of therapeutic vaccines. Rather than prevent infection, therapeutic vaccines would be designed to eliminate or ameliorate existing diseases, including chronic infectious diseases (e.g., those caused by herpes simplex virus (HSV), HIV, hepatitis B virus, hepatitis $\mathrm{C}$ virus, human papilloma virus, or $H$. pylori), tumors (e.g., melanoma, breast, or colon cancer), and allergic or autoimmune disorders (e.g., multiple sclerosis, type I diabetes, and rheumatoid arthritis). For example, a preliminary clinical study in subjects infected with HSV-2 showed a therapeutic benefit following vaccination with an adjuvanted recombinant vaccine ${ }^{105}$.

The level of toxicity acceptable for an adjuvant to be used in a therapeutic situation is likely to be higher than for a prophylactic vaccine designed to be used in healthy individuals, especially if the vaccine is designed to treat cancer or a life-threatening infectious disease. However, the acceptable safety profile for any new vaccine-adjuvant combination needs to be established in the clinic. Many adjuvants, including MPL ${ }^{106}, \mathrm{QS} 21^{21}$, and cytokines ${ }^{107}$, are currently being evaluated for the development of cancer vaccines.

Therapeutic vaccines may also be developed for mucosal administration, since an LT mutant has been shown to eradicate an established infection with $H$. pylori in mice ${ }^{108}$. In addition, preliminary studies indicate that oral administration of antigens can result in the amelioration of autoimmune diseases ${ }^{109}$.

\section{Future developments}

A desirable feature of an adjuvant is that it should specifically enhance the immune response to the vaccine antigen with which it is coadministered. An adjuvant with broad nonspecific effects has more potential for the induction of adverse immunological events. To achieve a specific effect, site-specific delivery, or adjuvant targeting may be advantageous. Targeting may be achieved at several different levels to include organ-specific delivery to local lymph nodes, cell-specific targeting to APCs within lymph nodes or in the peripheral tissues, or targeting to subcellular compartments (e.g., the proteosome to promote class I presentation or the nucleus for DNA vaccines).

Microparticles are "passively" targeted to MALT following mucosal delivery because of their particulate nature ${ }^{110}$. However, "active" targeting has also been achieved through the use of lectins, which have successfully targeted antigen $s^{111}$, liposomes ${ }^{112}$, and microparticles ${ }^{113}$ to the M cells of MALT. In addition, lectin targeting has also been used to enhance the extent of uptake of microparticles following oral delivery ${ }^{114}$. This is a potentially important approach, as it is clear that the extent of particle uptake is low and targeting may improve the efficiency of mucosal delivery of vaccines. Particulate adjuvants are also passively targeted to APCs following systemic immunization, as one of the natural roles of these cells is to recognize and process pathogen particles. Even so, more effective targeting to APCs may be achieved through the use of specific ligands. For example, the mannose receptor has recently been used to target liposomes to APCs ${ }^{115}$.

Further developments in the delivery of adjuvants will be achieved through the identification of specific receptors on APCs (e.g., the recently identified TLR), which are thought to provide a link between the innate and adaptive immune responses ${ }^{116}$. As described, CpGs have been shown to promote the activation and maturation of DCs ${ }^{27,28}$. Although CpGs are reportedly taken up by nonspecific endocytosis, their possible interaction with an intracellular receptor offers another opportunity for site-specific delivery ${ }^{117}$.

Agren et al. ${ }^{118,119}$ have described an approach for targeting APCs that involves coexpression of two linked proteins with a targeting component and an adjuvant signal. They created a gene fusion of the A1 subunit of CT and the immunoglobulin binding domain of protein A from Staphylococcus aureus ${ }^{118,119}$. The construct was shown to be a potent adjuvant, following both mucosal and systemic administration, because of its ability to both bind B cells and upregulate CD86 and costimulatory signals. In addition, the approach promoted B-cell proliferation and prevented apoptosis. This gene construct illustrates a new strategy for the targeted delivery of adjuvant activity to a selected group of cells. However, a possible limitation of this approach is that targeting of antigen to a specific subset of APCs (B cells) may not induce a sufficiently broad immune response. For example, it remains to be determined whether CTL responses are also induced by targeting to B cells.

An alternative approach to vaccine targeting for CTL induction has also been described using a fusion protein of antigen with a bacterial toxin to deliver the antigen specifically to the MHC class I processing pathway ${ }^{120}$. Targeting of adjuvants to a subset of APCs has also been achieved through the topical application of vaccines, which allows antigen to gain access to the Langerhans cells in the superficial layers of the skin ${ }^{103}$.

Future adjuvants will probably exploit more site-specific delivery systems for both mucosal and systemic administration. In addition, the identification of specific receptors on APCs is likely to allow targeting of adjuvants for the optimal induction of potent and specific immune responses. However, further developments in novel adjuvants will likely be driven by a better understanding of the mechanism of action of currently available adjuvants, and this is an area of research that requires much additional work.

\section{Acknowledgments}

We would like to acknowledge the contributions of our colleagues in Chiron Corporation to the ideas contained in this review, particularly Rino Rappuoli, John Donnelly, Margaret Liu, and Gary Ott. We would also like to thank Peter Anderson and Nelle Cronen for help in the manuscript preparation. We are grateful to Terry Ulrich and Charlotte Read-Kensil for the provision of clinical data on MPL and QS21 adjuvants, respectively.

1. Ramon, G. Sur la toxine et sur l'anatoxine diphtheriques. Ann. Inst. Pasteur 38,1-10 (1924).

2. Vogel, F.R. \& Powell, M.F. in A vaccine design: the subunit and adjuvant approach. (eds Powell, M.F. \& Newman, M.) 141-227 (Plenum Press, New York, NY; 1995).

3. Gupta, R.K. 1998. Aluminum compounds as vaccine. Advanced Drug Delivery Reviews 32,155-172 (1998).

4. Relyveld, E.H., Bizzini, B. \& Gupta, R.K. Rational approaches to reduce adverse reactions in man to vaccines containing tetanus and diphtheria toxoids. Vaccine 16, 1016-1023 (1998).

5. Edelman R. in New generation vaccines, 2nd Edn. (eds Levine, M.M., Woodrow, G.C., Kaper, J.B. \& Cobon, G.S.) 173-192 (Marcel Dekker Inc., New York, NY; 1997).

6. Cherwinski, H.M., Schumacher, J.H., Brown, K.D. \& Mosmann, T.R. Two types of mouse helper T cell clone. III. Further differences in lymphokine synthesis between Th1 and Th2 clones revealed by RNA hybridization, functionally mono- 
specific bioassays, and monoclonal antibodies. J. Exp. Med. 166, 1229-1244 (1987).

7. Mosmann, T.R. \& Moore, K.W. The role of IL-10 in cross-regulation of TH1 and TH2 responses. Immunoparasitol. Today A49-A53 (1991).

8. Romagnani, S. Human TH1 and TH2 subsets: doubt no more. Immunol. Today 12, 256-257 (1991).

9. Snapper, C.M. \& Paul, W.E. Interferon- $\gamma$ and B-cell stimulatory factor 1 reciprocally regulate Ig isotype production. Science 236, 944-947 (1987).

10. Poltorak, A. et al. Defective LPS signaling in $\mathrm{C} 3 \mathrm{H} / \mathrm{Hej}$ and $\mathrm{C} 57 \mathrm{BL} / 10 \mathrm{ScCr}$ mice: mutations in TIr4 gene. Science 282, 2085-2088 (1998)

11. Kirschning, C.J., Wesche, H., Ayres, T.M. \& Rothe, M. Human toll-like receptor 2 confers responsiveness to bacterial lipopolysaccharide. J. Exp. Med. 188, 2091-2097 (1998).

12. Yang, R.B. et al. Toll-like receptor-2 mediates lipopolysaccharide-induced cellular signalling. Nature 395, 284-288 (1998).

13. Gustafson, G.L. \& Rhodes, M.J. Bacterial cell wall products as adjuvants: early interferon gamma as marker for adjuvants that enhance protective immunity. Res. Immunol. 143, 483-488 (1992).

14. Ulrich, J.T. \& Ulrich, K.R. in Vaccine design: the subunit and adjuvant approach. (Powell, M. \& Newman, M.) 495-524 (Plenum Press, New York, NY; 1995).

15. Thoelen, S. et al. Safety and immunogenicity of a hepatitis B vaccine formulated with a novel adjuvant system. Vaccine 16, 708-714 (1998).

16. Sasaki, S. et al. Monophosphoryl lipid A enhances both humoral and cell-mediated immune responses to DNA vaccination against human immunodeficiency virus type 1. Infect. Immun. 65, 3520-3528 (1997).

17. Kensil, C.R. Saponins as vaccine adjuvants. Crit. Rev. Ther. Drug Carrier Syst. 13,1-55 (1996).

18. Soltysik S. et al. Structure/function studies of QS21 adjuvant: assessment of triterpene aldehyde and glucuronic acid roles in adjuvant function. Vaccine 13, 1403-1410 (1995)

19. Glaueri, A.M., Dingie, J.T. \& Lucy, J.A. Action of saponins on biological membranes. Nature 196, 953 (1962).

20. Kensil, C.R. \& Kammer, R. QS-21: a water-soluble triterpene glycoside adjuvant. Exp. Opin. Invest. Drugs 7, 1475-1482 (1998).

21. Livingston, P.O. et al. Phase I trial of immunological adjuvant QS-21 with a GM2 ganglioside-KLH conjugate vaccine in patients with malignant melanoma. Vaccine 12, 1275-1280 (1994).

22. Sasaki, S. et al. Induction of systemic and mucosal immune responses to human immunodeficiency virus type 1 by a DNA vaccine formulated with QS-21 saponin adjuvant via intramuscular and intranasal routes. J. Virol. 72, 4931-4939 (1998).

23. Tokunaga, T. et al. Antitumor activity of deoxyribonucleic acid fraction from Mycobacterium bovis GCG. I. Isolation, physiochemical characterization and antitumor activity. J. Natl. Cancer Inst. 72, 955-962 (1984).

24. Messina, J.P., Gilkeson, G.S. \& Pisetsky, D.S. Stimulation of in vitro murine lymphocyte proliferation by bacterial DNA. J. Immunol. 147, 1759-1764 (1991).

25. Krieg, A.M. et al. CpG motifs in bacterial DNA trigger direct B-cell activation. Nature 374, 546-549 (1995).

26. Bird, A.P. CpG islands as gene markers in the vertebrate nucleus. Trends Genet. 3, 342-347 (1987).

27. Jakob, T., Walker, P.S., Krieg, A.M., Udey, M.C. \& Vogel, J.C. Activation of cutaneous dendritic cells by CpG-containing oligodeoxynucleotides: a role for dendritic cells in the augmentation of Th1 responses by immunostimulatory DNA. J. Immunol. 161, 3042-3049 (1998).

28. Sparwasser, T. et al. Bacterial DNA and immunostimulatory CpG oligonucleotides trigger maturation and activation of murine dendritic cells. Eur. J. Immunol. 28, 2045-2054 (1998).

29. Davis, H.L. et al. CpG DNA is a potent enhancer of specific immunity in mice immunized with recombinant hepatitis B surface antigen. J. Immunol. 160, 870-876 (1998).

30. Sun, S., Kishimoto, H. \& Sprent, J. DNA as an adjuvant: capacity of insect DNA and synthetic oligodeoxynucleotides to augment $T$ cell responses to specific antigen. J. Exp. Med. 187, 1145-1150 (1998).

31. Moldoveanu, Z., Love-Homan, L., Huang, W.Q. \& Krieg, A.M. CpG DNA, a novel immune enhancer for systemic and mucosal immunization with influenza virus. Vaccine 16,1216-1224 (1998).

32. McCluskie, M.J. \& Davis, H.L. CpG DNA is a potent enhancer of systemic and mucosal immune responses against hepatitis B surface antigen with intranasal administration to mice. J. Immunol. 161, 4463-4466 (1998)

33. Klinman, D.M., Barnhart, K.M. \& Conover, J. CpG motifs as immune adjuvants. Vaccine 17, 19-25 (1999).

34. Broide, D. et al. Immunostimulatory DNA sequences inhibit IL-5, eosinophilic inflammation, and airway hyperresponsiveness in mice. J. Immunol. 161, 7054-7062 (1998)

35. Andrew, W.H. in Vaccine design: the subunit approach. (eds Powell, M.F. \& Newman, M.J.) 645-658 (Plenum Press, New Yok, NY; 1995).

36. Salgaller, M.L. \& Lodge, P.A. Use of cellular and cytokine adjuvants in the immunotherapy of cancer. J. Surg. Oncol. 68, 122-138 (1998)

37. Allison, A.C. \& Byars, N.E. An adjuvant formulation that selectively elicits the formation of antibodies of protective isotype and of cell-mediated immunity. $\mathrm{J}$. Immunol. Methods 95, 157-168 (1986).

38. Ott, G, Barchfeld, G.L. \& Van Nest, G. Enhancement of humoral response against human influenza vaccine with the simple submicron oil/water emulsion adjuvant MF59. Vaccine 13, 1557-1562 (1995).

39. Ott, G. et al. in Vaccine design: the subunit and adjuvant approach. (eds Powell, M.F. \& Newman, M.J.) 229-248 (Plenum Press, New York, NY; 1995).

40. O'Hagan, D.T., Ott, G.S. \& Van Nest, G. Recent advances in vaccine adjuvants: the development of MF59 emulsion and polymeric microparticles. Mol. Med. Today 3, 69-75 (1997).

41. Higgins, D.A., Carlson, J.R. \& Van Nest, G. MF59 adjuvant enhances the immunogenicity of influenza vaccine in both young and old mice. Vaccine 14, 478-484 (1996).

42. Cataldo, D.M. \& Van Nest, G. The adjuvant MF59 increases the immunogenicity and protective efficacy of subunit influenza vaccine in mice. Vaccine 15, 1710-1715 (1997)

43. Traquina, P., Morandi, M., Contorni, M. \& Van Nest, G. MF59 adjuvant enhances the antibody response to recombinant hepatitis $B$ surface antigen vaccine in primates. J. Infect. Dis. 174, 1168-1175 (1996).

44. Granoff, D.M., Mchugh, Y.E., Raff, H.V., Mokatrin, A.S. \& Van Nest, G.A. MF59 adjuvant enhances antibody responses of infant baboons immunized with Haemophilus influenzae Type $\mathrm{b}$ and Neisseria meningitidis group $\mathrm{C}$ oligosaccharide-CRM197 conjugate vaccine. Infect. Immun. 65, 1710-1715 (1997).

45. Dupuis, M. et al. Dendritic cells internalize vaccine adjuvant after intramuscular injection. Cell. Immunol. 186, 18-27 (1998).

46. Kahn, J.O. et al. Clinical and immunologic responses to Human Immunodeficiency Virus (HIV) Type I SF2 gp120 subunit vaccine combined with MF59 adjuvant with or without muramyl tripeptide dipalmitoyl phosphatidylethanolamine in non HIV-infected human volunteers. J. Infect. Dis. 170, 1288-1291 (1994).

47. Langenberg, A.G.M. et al. A recombinant glycoprotein vaccine for herpes simplex type 2: safety and immunogenicity. Ann. Intern. Med. 122, 889-898 (1995).

48. Ling, I.T. et al. Immunization against the murine malaria parasite Plasmodium yoelii using a recombinant protein with adjuvants developed for clinical use. Vaccine 15, 1562-1567 (1997).

49. Stoute, J.A. et al. A preliminary evaluation of a recombinant circumsporozoite protein vaccine against Plasmodium falciparum malaria. N. Engl. J. Med. 336, 86-91 (1996).

50. Gregoriadis G. Immunological adjuvants: a role for liposomes. Immunol. Today 11, 89-97 (1990).

51. Alving C.R. Immunologic aspects of liposomes, presentation and processing of liposomal protein and phospholipid antigens. Biochem. Biophys. Acta. 1113, 307-322 (1992)

52. Ambrosch, F. et al. Immunogenicity and protectivity of a new liposomal hepatitis A vaccine. Vaccine 15, 1209-1213 (1997)

53. Gould-Fogerite, S. et al. Targeting immune response induction with cochleate and liposome-based vaccines. Advanced Drug Delivery Reviews 32, 273 (1998).

54. Chen, H., Torchilin, V. \& Langer, R. Polymerized liposomes as potential oral vaccine carriers: stability and bioavailability. J. Controlled Release 42, 263-272 (1996).

55. Barr, I.G., Sjolander, A. \& Cox, J.C. Iscoms and other saponin based adjuvants. Advanced Drug Delivery Reviews 32, 247-272 (1998).

56. Rimmelzwaan, G.F. et al. Induction of protective immunity against influenza virus in a macaque model: comparison of conventional and iscom vaccines. J. Gen. Virol. 78, 757-765 (1997)

57. Smith, R.E., Donachie, A.M., Grdic, D., Lycke, N. \& Mowat, A.Mcl. Immune-stimulating complexes induce an IL-12 dependent cascade of innate immune responses. J. Immunol. 162, 5536-5546 (1999).

58. Okada, H. \& Toguichi, H. Biodegradable microspheres in drug delivery. CRC Crit. Rev. Ther. Drug Carrier Syst. 12, 1-99 (1995).

59. Putney, S.D. \& Burke, P.A. Improving protein therapeutics with sustained-release formulations. Nat. Biotechnol. 16, 153-157 (1998).

60. O'Hagan D.T. et al. Biodegradable microparticles as controlled release antigen delivery systems. Immunology 73, 239-242 (1991).

61. O'Hagan, D.T., Jeffery, H., Roberts, M.J.J., McGee, J.P. \& Davis, S.S. Controlled release microparticles for vaccine development. Vaccine 9, 768-771 (1991).

62. Eldridge, J.H., Stass, J.K., Meulbroek, J.A., Tice, T.R. \& Gilley, R.M. Biodegradable and biocompatible poly (DL-lactide-co-glycolide) microspheres as an adjuvant for staphylococcal enterotoxin B toxoid which enhances the level of toxin-neutralizing antibodies. Infect. Immun. 59, 2978-2986 (1991).

63. O'Hagan, D.T., Jeffery, H. \& Davis, S.S. Long term antibody responses in mice following subcutaneous immunization with ovalbumin entrapped in biodegradable microparticles. Vaccine 11, 965-969 (1993).

64. Maloy, K.J., Donachie, A.M., O'Hagan, D.T. \& Mowat, A.M. Induction of mucosal and systemic immune responses by immunization with ovalbumin entrapped in poly (lactide-co-glycolide) microparticles. Immunology 81, 661-667 (1994).

65. Moore, A. et al. Immunization with a soluble recombinant HIV protein entrapped in biodegradable microparticles induces HIV-specific CD8 ${ }^{+}$cytotoxic T lymphocytes and CD4 ${ }^{+}$Th1 cells. Vaccine 13, 1741-1749 (1995).

66. Nixon, D.F. et al. Synthetic peptides entrapped in microparticles can elicit cytotoxic T cell activity. Vaccine 14, 1523-1530 (1996).

67. Hedley, M.L., Curley, J. \& Urban, R. Microspheres containing plasmid-encoded antigens elicit cytotoxic T-cell responses. Nat. Med. 4, 365-368 (1998).

68. O'Hagan D.T. in New generation vaccines, 2nd Edn. (eds Levine, M.M., Woodrow, G.C., Kaper, J.B. \& Cobon, G.S.) 215-228 (Marcel Dekker Inc., New York, NY; 1997).

69. O'Hagan, D., Singh, M. \& Gupta, R.K. Poly(lactide-co-glycolide) microparticles for the development of single-dose controlled-release vaccines. Advanced Drug Delivery Reviews 32, 225-246 (1998)

70. Newman, M.J., Balusubramanian, M. \& Todd., C.W. Development of adjuvantactive nonionic block copolymers. Advanced Drug Delivery Reviews 32, 199-224 (1998).

71. Payne, L.G. et al. Poly[di(carboxylatophenoxy) phosphazene] (PCPP) is a potent immunoadjuvant for an influenza vaccine. Vaccine 16, 92-98 (1997)

72. Lang, J. Clinical trials for adjuvant evaluation. Nasal vaccine delivery conference (Management Forum, London, January 21-22, 1999).

73. Valenzuela, P., Medina, A., Rutter, W.J., Ammerer, G., \& Hall, B.D. Synthesis and assembly of hepatitis B virus surface antigen particles in yeast. Nature 298 347-350 (1982).

74. Schirmbeck, R. et al. Priming of class-I restricted cytotoxic T lymphocytes by vaccination with recombinant protein antigens. Vaccine 13, 857-865 (1995).

75. Gilbert, S.C. et al. A protein particle vaccine containing multiple malaria epitopes. Nat. Biotechnol. 15, 1280-1284 (1997).

76. Klavinskis, L.S. et al. Mucosal or targeted lymph node immunization of macaques with a particulate SIVp27 protein elicits virus-specific CTL in the genito-rectal mucosa and draining lymph nodes. J. Immunol. 157, 2521-2527 (1996). 
77. Martin, S.J. et al. Immunization of human HIV-seronegative volunteers with recombinant p17/p24:Ty virus-like particles elicit HIV-1 p24-specific cellular and humoral immune responses. AIDS 7, 1315-1323 (1993).

78. Levine, M.M. \& Dougan, G. Optimism over vaccines administered via mucosal surfaces. Lancet 351, 1375-1376 (1998).

79. O'Hagan D.T. in Novel delivery systems for oral vaccines. (ed. O'Hagan, D.T.) 175-205 (CRC Press, Boca Raton, FL; 1994).

80. Eldridge, J.H. et al. Controlled vaccine release in the gut associated lymphoid tissues. I. orally administered biodegradable microspheres target the Peyer's patches. J. Controlled Release 11, 205-214 (1990).

81. Challacombe, S.J., Rahman, D., Jeffery, H., Davis, S.S. \& O'Hagan, D.T. Enhanced secretory IgA and systemic IgG after oral immunization with biodegradable microparticles: Immunology 76, 164-168 (1992).

82. Challacombe, S.J., Rahman, D. \& O'Hagan, D.T. Salivary, gut, vaginal and nasal antibody responses after oral immunization with biodegradable microparticles. Vaccine 15, 169-175 (1997).

83. Jones, D.H. et al. Oral immunization with microencapsulated pertussis fimbriae induces protective immunity in mice. Infect. Immun. 64, 489-494 (1996).

84. Cahill, E.S. et al. Immune responses and protection against Bordetella pertussis infection after intranasal immunization of mice with filamentous haemagglutinin. Vaccine 13, 455-462 (1995).

85. Shahin, R., Leef, M., Eldridge, J.H., Hudson, M. \& Gilley, R. Adjuvanticity and protective immunity elicited by Bordetella pertussis antigens encapsulated in poly (D,L-lactide-co-glycolide) microspheres. Infect. Immun. 63, 1195-1200 (1995).

86. Whittum-Hudson, J.A., Ling-Ling, A., Saltzman, W.M., Prendergast, R.A. \& MacDonald, A.B. Oral immunization with an anti-idiotype antibody to the exoglycolipid antigen protects against experimental Chlamydia trachomatis infection. Nat. Med. 2, 1116-1121 (1996)

87. Allaoi-Attarki, K. et al. Protective immunity against Salmonella typhimurium elicited in mice by oral vaccination with phosphorylcholine encapsulated in poly (DLlactide-co-glycolide) microspheres. Infect. Immun. 65, 853-857 (1997)

88. Marx, P.A. et al. Protection against vaginal SIV transmission with microencapsulated vaccine. Science 260, 1323-1327 (1993)

89. Tseng, J. et al. Humoral immunity to aerosolized staphylococcal enterotoxin B (SEB), a superantigen, in monkeys vaccinated with SEB toxoid-containing microspheres. Infect. Immun. 63, 2880-2885 (1995).

90. Ugozzoli, M., O'Hagan, D.T. \& Ott, G.S. Intranasal immunization of mice with herpes simplex virus type 2 recombinant gD2: the effect of adjuvants on mucosal and serum antibody responses. Immunology 93, 563-571 (1998).

91. Morrell, C. et al. Biologically erodible microspheres as potential oral drug delivery systems. Nature 386, 410-414 (1997)

92. Jones, D.H., Corris, S., McDonald, S., Clegg, J.C.S. \& Farrar, G.H. Poly(DL-lactide-co-glycolide)-encapsulated plasmid DNA elicits systemic and mucosal antibody responses to encoded protein after oral administration. Vaccine 15 814-817 (1997).

93. Ando, S., Putnam, D., Pack, D.W. \& Langer, R. PLGA microspheres containing plasmid DNA: Preservation of supercoiled DNA via cryopreparation and carbohydrate stabilization. J. Pharm. Sci. 88, 126-130 (1998).

94. O'Hagan, D.T. The intestinal uptake of particles and the implications for drug and antigen delivery. J. Anat. 189, 477-482 (1996).

95. Douce, G. et al. Mutants of Escherichia coli heat labile enterotoxin lacking ADPribosyl transferase activity act as non-toxic mucosal adjuvants. Proc. Natl. Acad. Sci. USA 92, 1644-1648 (1995).

96. Douce, G., Fontana, M., Pizza, M, Rappuoli, R., \& Dougan, G. Intranasal immunogenicity and adjuvanticity of site-directed mutant derivatives of Cholera toxin. Infect. Immun. 65, 2821-2828 (1997).

97. Dickison, B.L. \& Clements, J.D. Dissociation of Escherichia coli heat-labile enterotoxin adjuvanticity from ADP-rybosyltranferase activity. Infect. Immun. 63, 1617-1623 (1995)

98. Di Tommaso, A. et al. Induction of antigen-specific antibodies in vaginal secretions by using a nontoxic mutant of heat labile enterotoxin as a mucosal adjuvant. Infect. Immun. 64, 974-979 (1996).
99. Giannelli, V. et al. Protease susceptibility and toxicity of heat-labile enterotoxins with a mutation in the active site or in the protease-sensitive loop. Infect. Immun. 65, 331-334 (1997).

100 Giuliani, M.M. et al. Mucosal adjuvanticity and immunogenicity of LTR72, a nove mutant of Escherichia coli heat-labile enterotoxin with partial knockout of ADPribosyltransferase activity. J. Exp. Med. 187, 1-10 (1998).

101 Marchetti, M. et al. Protection against Helicobacter pylori infection in mice by intragastric vaccination with $H$. pylori antigens is achieved using a non-toxic mutant of E. coli heat labile enterotoxin (LT) as an adjuvant. Vaccine 16, 33-37 (1998).

102 Rappuoli, R., Pizza, M., Douce, G. \& Dougan, G. A relationship between the structure and function of cholera and Escherichia coli heat labile enterotoxins and their immunological activity at mucosal surfaces. Immunol. Today, (1999).

103 Glenn, G.M., Rao, M., Matyas, G.R. \& Alving, C.R. Skin immunization made possible by cholera toxin. Nature 391, 851 (1998).

104 Tacket, C.O. et al. Immunogenicity in humans of a recombinant bacterial antigen delivered in a transgenic potato. Nature 4, 607-609 (1998).

105 Straus, S.E. et al. Placebo-controlled trial of vaccination with recombinant glycoprotein $\mathrm{D}$ of herpes simplex virus type 2 for immunotherapy of genital herpes. Lancet 343, 1460-1463 (1994).

106 Longenecker, B.M., Reddish, M., Koganty, R. \& MacLean, G.D. Immune responses of mice and human breast cancer patients following immunization with synthetic Sialyl-Tn conjugated to KLH plus detox adjuvant. Ann. N.Y. Acad. Sci. 690, 276-291 (1993)

107 Agrawal, B., Krantz, M.J., Reddish, M.A. \& Longenecker, B.M. Cancer-associated MUC1 mucin inhibits human T-cell proliferation, which is reversible by IL-2. Nat. Med. 4, 43-49 (1998).

108 Ghiara, P. et al. Therapeutic intragastric vaccination against Helicobacter pylori in mice eradicates an otherwise chronic infection and confers protection against reinfection. Infect. Immun. 65, 4996-5002 (1997).

109 Weiner, H.L. Oral tolerance: Immune mechanisms and treatment of autoimmune diseases. Immunol. Today 19, 335-343 (1997)

110 O'Hagan, D.T. Microparticles and polymers for the mucosal delivery of vaccines. Adv. Drug Deliv. Rev. 34, 305-320 (1998).

111 Giannasca, P.J., Boden, J.A. \& Monath, T.P. Targeted delivery of antigen to hamster nasal lymphoid tissue with M-cell-directed lectins. Infect. Immun. 65, 4288-4298 (1997).

112 Chen, H., Torchilin, V. \& Langer, R. Lectin-bearing polymerized liposomes as potential oral vaccine carriers. Pharm. Res. 13, 1378-1383 (1996).

113 Foster, N., Clark., M.A., Jepson, M.A. \& Hirst, B.H. 1997. Ulex europaeus 1 lectin targets microspheres to mouse Peyer's patch M-cells in vivo. Vaccine 16, 536-541 (1996).

114 Hussain, N., Jani, P.U. \& Florence A.T. Enhanced oral uptake of tomato lectinconjugated nanoparticles in the rat. Pharm. Res. 14, 613-618 (1997).

115 Toda, S. et al. HIV-1-specific cell-mediated immune responses induced by DNA vaccination were enhanced by mannan-coated liposomes and inhibited by antiinterferon- $\gamma$ antibody. Immunology 92, 111-117 (1997).

116 Medzhiton, R., Preston-Hurlbut, P. \& Janeway, C.A. Jr. A human homologue of the Drosophila toll protein signals activation of adaptive immunity. Nature $\mathbf{3 8 8}$ 394-397 (1997).

117 Hacker, $H$. et al. CpG-DNA-specific activation of antigen-presenting cells requires stress kinase activity and is preceded by non-specific endocytosis and endosomal maturation. EMBO J. 17, 6230-6240 (1998).

118 Agren, L.C., Ekman, L., Lowenadler, B. \& Lycke, N.Y. Genetically engineered nontoxic vaccine adjuvant that combines $B$ cell targeting with immunomodulation by cholera toxin A1 subunit. J. Immunol. 158, 3936-3946 (1997).

119 Agren, L.C., Ekman, L., Lowenadler, B., Nedrud, J.G. \& Lycke, N.Y. Adjuvanticity of the cholera toxin A1-based gene fusion protein, CTA1-DD, is critically dependent on the ADP-ribosyltransferase and Ig-binding activity. J. Immunol. 162, 2432-2440 (1999).

120 Goletz, T.J. et al. Targeting HIV proteins to the major histocompatibility complex class I processing pathway with a novel gp120-anthrax toxin fusion protein. Proc. Natl. Acad. Sci. USA 94, 12059-12064 (1997). 